\title{
Aspects phonologiques du français haïtien : influence du créole haïtien
}

\author{
Renauld Govain ${ }^{1}$
}

Faculté de Linguistique Appliquée, Université d'État d'Haïti, 38 rue Dufort, Port-au-Prince, Haïti

\begin{abstract}
Résumé. Le français haïtien (FH), caractérisé par des normes endogènes issues du contact du français avec le créole haïtien $(\mathrm{CH})$ - mais aussi, dans une certaine mesure, avec l'anglais et l'espagnol - et des spécificités locales diverses, est une variété de parler francophone propre à Haïti et différente à bien des égards des autres variétés de français. Ses spécificités se manifestent aux niveaux lexicosémantique, phonologique et, dans une moindre mesure, morphosyntaxique. À part Pompilus (1961) et Govain $(2009,2013)$ qui l'ont toutefois effleuré, l'aspect phonologique du FH n'est guère étudié. C'est cet aspect phonologique dans sa dimension synchronique qui nous intéresse dans cette contribution qui décrit brièvement les spécificités du FH en dégageant les influences du CH. Parmi les éléments que nous traiterons, signalons, au niveau des consonnes: des réalisations spécifiques de $/ \mathrm{n} /$ et de $/ \mathrm{b} /$, le maintien de $/ \mathrm{h} /$ dit aspiré, la (non)réalisation des consonnes branchantes en coda finale. Au niveau des voyelles : le maintien de l'opposition $/ \mathrm{e} / \sim / \varepsilon /$ dans toutes les positions et de l'opposition $/ \tilde{\varepsilon} /$ $\sim / \tilde{\mathfrak{e}} /$, l'absence de l'opposition /a/ /a/, l'apparition selon le contexte d'articulation des glides $/ \mathrm{j} /$ ou $/ \mathrm{w} /$ (ou même d'un $/ \mathrm{s} /$ dans des contextes spécifiques) entre deux voyelles contiguës pour éviter le hiatus.
\end{abstract}

\begin{abstract}
Phonological aspects of Haitian French: influence of Haitian Creole. Haitian French (HF) is a variety of French speaking specific to Haiti and different in many ways from other varieties of French. characterized by endogenous norms which result from the contact of French with the Haitian Creole (HC) - but also, to a certain extent, with English and Spanish - and many local specificities. Its specificities can manifest at the lexico-semantic, phonological and, in a least measure, morphosyntactic aspects. Except for Pompilus (1961) and Govain $(2009,2013)$ who have approached it, the phonological aspect of $\mathrm{HF}$ is not much studied. This contribution is interested in this phonological aspect in its synchronic dimension. It briefly describes the specificities of HF while highlighting the influences of HC. Among the elements that we will deal with, we can point out, at the consonantal level: specific realizations of $/ \mathrm{n} /$ and $/ \mathrm{b} /$, the use of $/ \mathrm{h} /$ said aspirated, the (non-)realization of branching consonants in final coda. At the level of vowels: maintaining the /e/ $/ \varepsilon /$ opposition in all positions and the $/ \tilde{\varepsilon} / \sim / \tilde{\mathfrak{e}} /$ opposition, the absence of the $/ \mathrm{a} /$ $\sim / \mathrm{a} /$ opposition, the appearance according to the context of articulation of the glides $/ \mathrm{j} /$ or $/ \mathrm{w} /$ (or even $\mathrm{a} / \mathrm{b} /$ in specific contexts) between two contiguous vowels to avoid hiatus.
\end{abstract}

\footnotetext{
${ }^{1}$ renauld.govain@ueh.edu.ht
} 


\section{Introduction}

Le français pratiqué en Haïti n'est pas en tout point identique à celui parlé dans le reste de la francophonie. Arrivé en Haïti au milieu de la première moitié du XVII ${ }^{\mathrm{e}}$ siècle, le français va évoluer en se différenciant d'autres variétés de parlers francophones et développer ce qu'il convient d'appeler le français haïtien (Pompilus 1961 ; Fattier 2010 ; Saint-Fort 2007 ; Govain 2009, 2013) qui se caractérise par des normes endogènes (Govain 2008) issues de spécificités locales diverses et du contact de langues, où le français et le créole haïtien $(\mathrm{CH})$ s'influencent mutuellement. Le français haïtien $(\mathrm{FH})$ est dès lors une variété de parler francophone propre à Haïti et différente à bien des égards des autres variétés de français, la variété étant une forme linguistique relevant d'une langue dite de référence et propre à une région, un groupe d'individus en rapport avec la profession, l'âge, des facteurs socioculturels, écologiques, etc. (Govain 2013). La conscience de l'existence du FH semble avoir émergé relativement tôt, le romancier haïtien Fernand Hibbert a fait dire en 1923 à son personnage Gérard Delhi : «... par l'action du milieu, le français que nous parlons et écrivons n'est pas plus le français de France que l'anglais des États-Unis n'est l'anglais des Iles britanniques » (Hibbert 1993 : 77). Les spécificités du FH se manifestent aux niveaux lexico-sémantique, phonologique et, dans une moindre mesure, morphosyntaxique. L'aspect phonologique du FH n'est guère étudié, Pompilus (1961) et Govain (2009, 2013) l'ont toutefois effleuré. C'est cet aspect dans sa dimension synchronique qui nous intéresse ici.

Cette contribution décrit brièvement les spécificités du FH en passant par la mise en regard des convergences phonologiques entre le $\mathrm{FH}$ et le $\mathrm{CH}$. Les données du corpus proviennent d'une enquête consistant en l'enregistrement de productions d'étudiants de $1^{\mathrm{e}}$ année d'Université, de l'enregistrement de productions de locuteurs notamment à la radio et à la télévision et d'une enquête consistant en la lecture enregistrée d'un texte d'une durée moyenne de 90 secondes. Nous avons retenu 10 lectures. Les lecteurs, dont deux femmes, âgés de 25 à 65 ans, ont tous fait l'enseignement supérieur (de la licence au doctorat). Le mieux aurait été d'enregistrer des échanges naturels de locuteurs. Mais, en Haïti, le français n'est pas une langue de communication courante, excepté dans des situations hautement formelles et officielles.

Pour marquer la différence entre le $\mathrm{FH}$ et le français dit de référence (FR), nous comparons la forme acoustique sonore variable produite dans le discours effectif des locuteurs et la forme mentale, stable, invariable (Darcy 2006) telle que le dictionnaire en propose la réalisation phonétique. La forme dite de référence correspond à cette forme mentale. Parmi les éléments que nous traiterons ici, signalons, au niveau des consonnes : des réalisations spécifiques de $/ \mathrm{n} /$ et de $/ \mathrm{b} /$, le maintien de $/ \mathrm{h} /$ dit aspiré, la (non-)réalisation des consonnes en coda branchante finale. Au niveau des voyelles : le maintien de l'opposition /e/ $\sim / \varepsilon /$ dans toutes les positions et de $/ \tilde{\varepsilon} / \sim / \tilde{\mathfrak{e}} /$, l'absence de l'opposition $/ \mathrm{a} / \sim / \mathrm{a} /$, l'apparition selon le contexte d'articulation des glides antihiatique $/ \mathrm{j} /$ ou $/ \mathrm{w} /$ (ou même d'un /в/ dans des contextes spécifiques) entre deux voyelles contiguës pour éviter le hiatus.

\section{Les spécificités}

Le FH possède théoriquement les mêmes phonèmes que le FR, excepté que /a/ antérieur n'y a

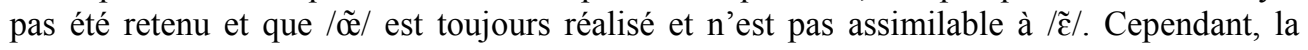
réalisation de certaines consonnes est parfois différente même si leurs distributions restent en fait les mêmes dans les deux variétés.

\subsection{Le phonème $/ \mathrm{h} /$}

$/ \mathrm{h} /$ est réalisé de manière fortement aspirée en $\mathrm{FH}$, comme dans *hache, *hamac, *hanche, *hantise, *hauteur, *héler, la ville de *Hinche, etc. L'école haïtienne enseigne à bien réaliser ce phonème en général lorsqu'il est dit aspiré. 9 des 10 lecteurs ont réalisé [haJ] pour 'hache', $[h \varepsilon]$ pour 'hait'. Celui qui a réalisé $[\mathrm{a} J]$ et $[\varepsilon]$, trentenaire, a étudié en Belgique 
francophone du master au doctorat dans le domaine de la sismologie. Cependant, il a réalisé le yod à la place de $/ \mathrm{h} /$ à l'origine dit aspiré dans dehors : [дəјəь]. Un lecteur dans la soixantaine (professeur d'anglais) a réalisé [дəһəь].

\subsection{Le phonème $/ \mathrm{n} /$}

En position interne, les locuteurs réalisent généralement $/ \mathrm{h} /$ à peu près comme en $\mathrm{FR}: 7$ lecteurs ont réalisé [salanak] 'Salagnac', 7 [senœ: ${ }^{\mathrm{r}}$ ] 'seigneur'. Mais, certains le réalisent comme le yod en cette position : 3 ont réalisé [salajak], 3 [sejœ(ь)]. Cependant, en dehors d'une vérification expérimentale, on peut être amené à croire que la réalisation que nous percevons ici comme $/ \mathrm{n} /$ peut être la combinaison des segments $/ \mathrm{n}+\mathrm{j} /$ notamment en position interne. En coda, les locuteurs le réalisent le plus souvent comme le yod $/ \mathrm{j} /: 4$ lecteurs ont réalisé [bว:j], 6 [ [a(ь)lmaj] 'Charlemagne', etc. À la suite de /i/ en finale de mot, la réalisation de $/ \mathrm{n} /$ rappelle généralement le yod, mais on observe une nasalisation de $/ \mathrm{i} /$ qui le précède : vigne [vĩj], signe [sĩj]. Cependant, dans ligne, il se réalise sous la forme de la vélaire $/ \mathrm{y} /: / \mathrm{lin} />$ [lĩn], évidemment, on sent aussi la portée nasale sur le /i/.

En général, les locuteurs réalisent normalement le phonème vélaire $/ \mathrm{y} /$, comme en anglais. 9 sur 10 ont réalisé le mot camping comme [kãmpin]. L'autre locuteur a renforcé la vélaire nasalisée de la gutturale à la fin : [kãmping]. Il est peut-être intéressant de noter que le /ãa nasal fait intervenir un [m] avant le /p/ de / kãpin/ sans toutefois remplacer le /p/ en question.

\subsection{Le phonème / $\mathbf{b} /$}

/ $\mathrm{s} /$ présente une réalisation en $\mathrm{FH}$ qui est quelque peu différente de la manière dont il est réalisé en français de la France continentale. Par exemple, Pompilus (1961) croit que la faiblesse articulatoire qui l'affecte est plus prononcée que celle des autres consonnes et qu'il est plutôt une fricative sonore vélaire, moins profonde que le $r$ français et très proche de la semi-consonne $w$. Il est certes difficile de le caractériser de manière indiscutable en dehors de laboratoire. Mais nos observations nous conduisent à y voir une fricative uvulaire en FH. Il peut être diversement noté selon ses réalisations et les langues. Il est donc protéiforme. Un ordre d'idées peut être donné dans Akpossan (2015) et, notamment, dans Navarro (2016). L'Alphabet phonétique international (API) offre les symboles suivants pour ses différentes réalisations (tiré de Navarro $(2016: 10)$ ).

$\begin{array}{cc}\text { Symbole API } & \text { Mode d'articul. } \\ \text { R } & \text { Vibrante } \\ \text { I } & \text { Battue } \\ \text { I } & \text { Approximante } \\ \text { I } & \text { Battue } \\ \text { I } & \text { Approximante } \\ & \\ \text { R } & \text { Vibrante } \\ \text { K } & \text { Fricative } \\ \text { I } & \text { Battue (latérale) }\end{array}$

Lieu d'articul.
Alvéolaire
Alvéolaire
Alvéolaire
Post-alvéolaire
Post-alvéolaire
(rétroflexe)
Uvulaire
Uvulaire
Alvéolaire

Navarro (op. cit.) souligne que la diversité de ses réalisations d'un point de vue fonctionnel est encore plus vaste et que la notation de la plupart d'entre elles n'est pas prévue dans le cadre de l'API et qu'on est obligé de recourir à des diacritiques pour les représenter.

En général, devant les voyelles labiales (/y, o, õ, u, ॰, , œ, œ̃/) les locuteurs haïtiens réalisent / / / sous la forme de $[\mathrm{w}]$ : problème [pwoblem], prudent [pwydã], propre [pwวp(в)], front [fwõ], preuve [pwœv], hébreux [ebwø], prune [pwyn], emprunt [ãpwõ], etc. Le trait labial de la voyelle qui suit le /ь/ participe de cette labialisation. Tous les 
exemples pris ici en considération présentent un contexte renforçant la labialisation de $/ \mathrm{s} /$ : en plus du caractère labial des voyelles en question, / / / est précédé d'une consonne labiale. Mais la condition première de cette labialisation est la présence de la voyelle labiale. Avec une consonne non labiale à gauche, la labialisation aura lieu. 7 des lecteurs ont réalisé [twote] pour trotter et [kwoke] pour croquer, 8 [pwopwate] pour propreté et [øwəp] pour Europe, 6 [ñ̃bwø] pour nombreux, 7 [3iwวf(l)] pour girofle, 6 [fwomaz] pour fromage, etc.

En effet, /b, p, f, v/ étant labiales, elles sont plus proches de /w/ que de /s/. Ainsi, /s/ précédé de l'une de ces consonnes peut se voir attribuer une réalisation labiale dans le parler de certains Haïtiens peu importe la nature de la voyelle qui suit (labiale ou non). C'est une forme d'harmonie labiale. Cette réalisation de /s/, dans ce contexte précis, s'observe notamment chez des locuteurs du nord d'Haïti. D'où les exemples suivants produits par deux étudiants originaires du nord (de première année de licence à l'Université d'État d'Haïti) :

[məsjø zə ne pa kə̃pwi la fwaz] </məsjø зə ne pa kə̃рьi la fьaz/

'Monsieur, je n'ai pas compris le phrase'

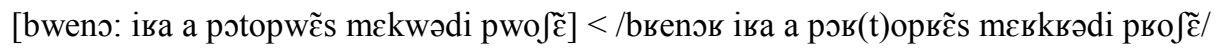

'Brénor ira à Port-au-Prince mercredi prochain'

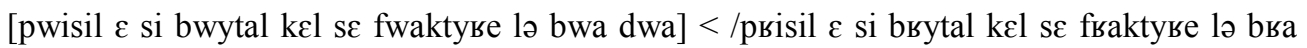
dвwa/

'Priscille est si brutale qu'elle s'est fracturé son bras droit'

On pourrait voir en $[\mathrm{w}]$ une certaine réalisation contextuelle de / $\mathbf{b} /$, mais il est évident que le trait labial de la voyelle qui suit ou de la consonne qui précède participe de cette labialisation. /в/ et [w] fonctionnent ainsi en distribution complémentaire. Le choix de l'une et de l'autre obéit à une contrainte liée au lieu d'articulation des voyelles environnantes : [w] intervient en environnement labial et / $\mathbf{~} /$ conserve sa réalisation dans les autres contextes. Ainsi, on n'entendra aucun locuteur prononcer *[patwi] pour patrie, *[meskinwi] pour mesquinerie, *[twavaj] pour travail, *[kwik] pour cric, ou encore *[ре(к)dwi] pour perdrix, etc. Il existe ainsi deux /w/ en FH : un /w/ sous-jacent comme dans [пуав] noir ou [tьwa] trois et un $[\mathrm{w}]$ qui est une réalisation contextuelle de / $/$ / comme dans les exemples cidessus.

En $\mathrm{FH}$, en général, /в/ suivi du groupe phonique /wa/, s'amuït pour ne laisser apercevoir que le /w/ : /ьwa/ > [wa] (roi), /dьwa/ > [dwa] (droit), /fьwa/ > [fwa] (froid), /кьшаь/ > [кwав] (сroire). On comprend facilement le fait que les deux sons se confondent dans ce contexte car ils sont confondus dans certains contextes comme nous l'avons déjà montré.

En coda de syllabe intérieure ou entre deux mots partageant une frontière syllabique, /ь/ n'est guère réalisé par certains locuteurs : 7 lecteurs ont réalisé [pu finis] pour pour finir, 5 [вәgad] pour regarde, 4 [а patis] pour à partir, 3 [t๕m] pour terme, 6 [Jalmaj] pour Charlemagne, 6 [adammã] pour ardemment, 1 [adamã] pour ardemment, etc. Walker (1984) établit le même constat pour ce qu'il appelle le français canadien. En coda finale, la plupart du temps, /ь/ se trouve sinon amuï, du moins affaibli et les locuteurs allongent la voyelle formant le noyau de la syllabe dont / $/ \mathrm{s} /$ est la coda et cet allongement tient lieu de sa réalisation. Ainsi, pour Bonjour Sœur Elvire, des locuteurs réalisent [bõzu: ${ }^{\mathrm{r}}$ sœ: ${ }^{\mathrm{r}}$ elvi: ${ }^{\mathrm{r}}$ ] ou pour " $A$ u revoir monsieur le professeur » on entend [о вәvwa: ${ }^{\mathrm{r}}$ məsjø lə pwofesœ: $\left.{ }^{\mathrm{r}}\right]^{\mathrm{i}}$. Par contre, quand / $/ \mathrm{s} /$ est prononcé dans ce contexte, il l'est de manière exagérée et cela témoigne de l'hypercorrection (Francard 1997) qui dévoile une stratégie consciente d'appropriation d'une légitimité linguistique et sociale. Ainsi, ils «outrepassent ceux du groupe le plus élevé dans leur tendance à employer les formes réputées correctes et appropriées au style soigné » (Labov 1976 : 193). Ils vont au-delà de ce qui est attendu, en réalisant le / $\mathrm{b} /$ de manière relativement roulée. On rencontre cette forme de prononciation du /в/ chez des journalistes, des animateurs de radio, des professeurs, des professionnels du 
droit, des hommes politiques, des pasteurs, des prêtres, etc.

\subsection{Effacement des consonnes branchantes finales}

Dans une coda branchante finale, les locuteurs prononcent en général la tête de la branchante et omettent la (les) branchante(s) : journalisme ou journaliste $>$ [зu(ь)nalis] (donc en dehors du contexte énonciatif, difficile de savoir si on parle du métier ou du professionnel), 4 lecteurs ont réalisé [3iwof] 'girofle', 2 [semes] 'semestre', 2 [ekes] 'équestre', 2 [egzis] 'existe', 4 [3ys] 'juste', 2 [õk] 'oncle', 4 [minis] et 1 [minist] 'ministre',

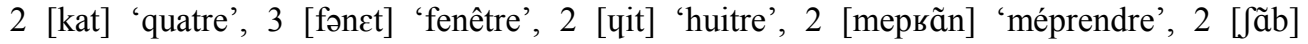
'chambre', etc.

D'un autre côté, il est remarqué une tendance à la nasalisation des codas branchantes $/ \mathrm{bs} /, / \mathrm{gs} /, / \mathrm{dr} /, / \mathrm{bl} /, / \mathrm{gl} /$ précédées d'une voyelle nasale $(\tilde{\mathrm{V}})$ en finale de mot. Dans ce cas, deux règles successives s'appliquent : 1 . l'effacement de la rhotique ou de la latérale ; 2 . l'assimilation progressive par nasalisation où les occlusives $/ \mathrm{b}, \mathrm{d}, \mathrm{g} / \mathrm{se}$ laissent nasaliser par

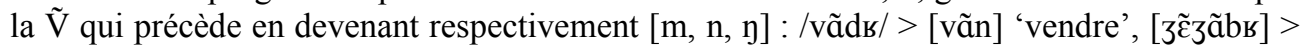

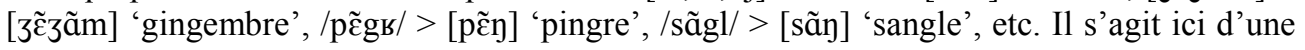
forme d'économie. La plupart des lecteurs du texte appliquent la première règle mais ne vont pas jusqu'à l'assimilation de nasalisation, les mots susceptibles d'être nasalisés dans ce contexte étant employés de manière isolée mais n'étant pas ancrés dans une dynamique de coarticulation dans une phrase. On peut toutefois rappeler que deux d'entre eux ont réalisé [терьãn] pour /mерьãdь/. Sur un plan formel, cette expérience éloigne le FH du français de la France continentale qui ne tolère pas qu'une $\tilde{C}$ suive une $\tilde{V}$ dans un mot. Mais, cette expérience articulatoire n'est sans doute pas spécifique au FH. Elle doit aussi se rencontrer dans d'autres variétés de FR. Walker (1984) l'a signalé pour le français canadien. Il ne serait pas surprenant qu'elle puisse être observée dans des productions de locuteurs de la France continentale en dépit de ce qui vient d'en être dit.

\subsection{Réalisation des géminées}

De manière générale, les Haïtiens ne réalisent pas la géminée. La gémination consiste en le renforcement d'une articulation consonantique qui en prolonge la durée et augmente l'intensité. La consonne géminée est alors plus longue, plus énergique qu'une consonne simple. La longueur est telle qu'on a l'impression qu'elle se scinde en deux en faisant percevoir la première comme plus implosive et la seconde plus explosive, que chaque partie appartient à une syllabe différente. Néanmoins, lorsqu'il s'agit des adverbes de manière en '-ment', les locuteurs la réalisent normalement. Ainsi, pour les adverbes récemment, ardemment et notamment, tous les lecteurs ont réalisé respectivement: [веsammã], [авdammã] et [notammã], excepté un seul (34 ans, master en science de l'éducation) qui a dit [adamã]. Mais aucune gémination n'est remarquée dans la réalisation phonétique de tellement.

Après /i/ la plupart des locuteurs font souvent entendre la gémination avec /1/, /m/, /n/

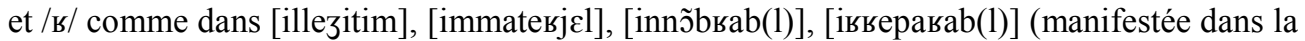
longueur observée dans la réalisation $\mathrm{du} / \mathrm{s} /$ ), pour illégitime, immatériel, innombrable, irréparable.

\section{Au niveau des voyelles}

\subsection{Deux voyelles contiguës à l'intérieur d'un mot}

En FH, la rencontre de deux voyelles est évitée au profit d'une épenthèse (métaplasme consistant en l'apparition à l'intérieur d'un mot d'un phonème non étymologique). Le locuteur sépare les deux voyelles par les antihiatiques $/ \mathrm{j} /$ ou $/ \mathrm{w} /$ suivant le contexte 
articulatoire : dehors [дәјов] ${ }^{\mathrm{ii}}$, géographie [зешоgьаfi], Noé [nоје], créole [kьејэl], cruel [kьујєl], linéaire [linejєь], Bethléem [betlejєm], en haut [ãwo], etc. Dans le cas de [dəјоь], le glide qui était attendu, en réalité, c'est le /w/, considérant l'aspect labial de la voyelle de droite. On peut considérer que la voyelle de gauche, en principe le schwa, neutre du point de vue articulatoire, possède une caractéristique de labiale. En effet, le schwa doit normalement être réalisé dans ce contexte notamment par rapport au son [h] à l'origine dit aspiré qui existe dans la conscience phonologique du locuteur francophone haïtien. Considérant que certains locuteurs (par exemple, ici 4 lecteurs) réalisent [дејəь] - avec [e] et non /ə/ - il est probable que le yod tienne à ce que dans la conscience phonologique des locuteurs il s'actualise /e/ et non /ə/. En effet, 4 lecteurs ont réalisé [dejo: ${ }^{\mathrm{r}}$ ], 1 [dehəь] (forme qui s'entend aussi au Québec (Meney 2017)) et les 5 autres [dəjo: ${ }^{r}$ ].

Dans l'environnement de /a/, intervient généralement $/ \mathrm{b} /$ qui est susceptible de se réaliser [w] devant une voyelle labiale : /kanaã/ $>$ [kanasã] 'Canaan' : 7 sur les 10 lecteurs

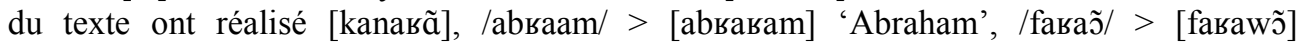

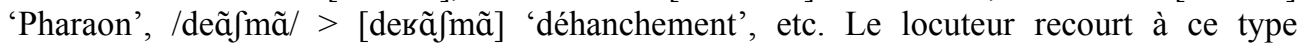
d'épenthèse dans le souci d'un adoucissement articulatoire, le créole, langue première des Haïtiens, ne comportant pas de mots avec deux voyelles contiguës.

En outre, certains locuteurs introduisent une diérèse dans la syllabe /-sja-/ à l'intérieur de mots en le faisant devenir [-sija-] comme dans /asosjasj̃̃/ > [asosijasjõ] 'association', /рьопว̃sjasjõ/ > [pwonõsijasjõ] 'prononciation', etc. 6 lecteurs ont réalisé ces deux mots avec la diérèse.

La synérèse est aussi observée dans certains mots où des locuteurs réalisent deux syllabes sous la forme d'une seule :/nocl/ $>$ [nwel] (6 des lecteurs ont réalisé ainsi) ou [nowعl] (réalisé ainsi par 4 des lecteurs) pour 'noël' (cette dernière réalisation s'observe aussi en Wallonie, en particulier dans l'est et le centre (Pierret 1994)), /luãz/ > [lwãz] ou [luwãz] 'louange' : sur les 10 lecteurs 5 ont réalisé [lwãz], 3 [luwãz] et 2 [lusãz]. Warnant (1996) signale, dans sa préface, l'intervention d'un glide dans les mêmes conditions en français. Parmi ses exemples, on retrouve justement /nozl/ > [nwعl] 'noël' et /kreasjã/ > [kвеjasjõ] 'création'. Mais il précise que la prononciation avec le glide n'appartient pas au français dit standard.

\subsection{L'opposition $/ \mathrm{e} / \sim / \varepsilon /$}

/e/ et $/ \varepsilon /$ conservent leur prononciation originelle en FH peu importe leur position dans un mot. Si dans certaines communautés comme en France continentale, l'opposition $/ \mathrm{e} / \sim / \varepsilon / \mathrm{se}$ neutralise au profit de [e] en finale de mot (même avec la présence d'une consonne de liaison), elle fonctionne normalement en $\mathrm{FH}$ où /e/ se réalise toujours [e] et / $/ \varepsilon /$ toujours $[\varepsilon]$ peu importent leurs positions dans un mot. Ainsi, en FH, il n'y a aucune confusion entre les mots dé et des, piqué et piquet, apprenez et apprenais car leur signifiant est à chaque fois

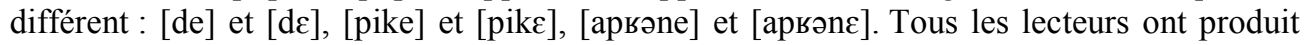

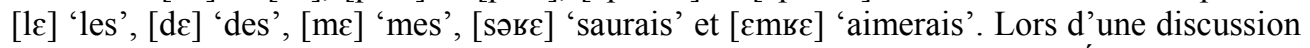
avec des étudiants de $3^{\mathrm{e}}$ année de licence de linguistique à l'Université d'État d'Haïti, un collègue enseignant français en mission en Haïti dit [lеfьь fe le fэь]. L'un des étudiants lui dit : « Votre phrase parait tautologique Monsieur. Comment [lеfэь fe le fəь] ? ». Le collègue a dû faire beaucoup d'effort pour expliquer qu'il a voulu dire «L'effort fait les forts ». D'où un exemple de différence entre l'écrit et l'oral dans la pratique de la langue. C'est ce que Gadet (2007) appelle une variation diamésique. Car la forme écrite est la même pour l'étudiant et l'enseignant, mais la réalisation est différente de l'un à l'autre. L'étudiant

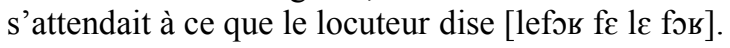

Nous savons, par ailleurs, que $[\varepsilon]$ se présente parfois comme une réalisation contextuelle de /e/, /e/ se réalisant [e] en syllabe ouverte et $[\varepsilon]$ en syllabe fermée [ete] $\sim$ [Ets] 'été $\sim$ être', [3əte] $\sim$ [3et] 'jeter $\sim$ jette' ou encore $[$ eple] $\sim$ [epsl] 'épeler $\sim$ épèle'. Ceci est valable tant pour le FR que pour le FH. 
Signalons au passage que 6 des 10 lecteurs ont réalisé le mot têtière comme [tzt $\left.{ }^{\top} \varepsilon:{ }^{\mathrm{r}}\right]$ avec un 'e' ouvert dans la première syllabe et non avec un 'e' fermé. Cette réalisation doit tenir au fait que 1) le mot provient de tête et 2) graphiquement, le graphème en question possède un accent circonflexe comme dans tête dont il est dérivé. Comme en français québécois, ces 6 locuteurs gardent la voyelle ouverte de la base dérivationnelle tête. Le dictionnaire du CNRTL (Cf. : https://www.cnrtl.fr/definition/têtière) propose les deux prononciations. Le Petit Robert propose la prononciation avec la voyelle ouverte. La prononciation avec la voyelle fermée en FR doit être un phénomène relativement récent.

\subsection{L'opposition /o/ /O/}

L'opposition /o/ / / / fonctionne, comme dans le cas de /e/ $/ \varepsilon /$, quasiment pareil en FR qu'en FH. Elle fonctionne en distribution complémentaire, /o/ se réalise [o] en syllabe ouverte et $[0]$ en syllabe fermée : [tвоt] $\sim$ [tвоte] 'trotte $\sim$ trotter' (la réalisation attendue en FH pour ces mots est plutôt [twot] $\sim$ [twote]), : [glob] $\sim$ [global] 'globe $\sim$ global' ou encore : [vol] $\sim$ [vole] 'vole $\sim$ voler'. Si de manière générale, les oppositions $/ \mathrm{e} / \sim / \varepsilon /$ et $/ \mathrm{o} / \sim / \mathrm{\jmath} /$ fonctionnent quasi-pareillement en FR et en FH, les francophones du nord d'Haïti respectent le plus souvent le fonctionnement en distribution complémentaire. Aussi réalisent-ils, comme il a été observé dans Govain (2009) : [lektyк] 'lecture', [عspкi] 'esprit', [testamã] 'testament'; [doktoва] 'doctorat', [moske] 'mosquée', [kostym] 'costume', etc. Il est aussi à remarquer que lorsque /e/ correspond à la graphie 'ai' (excepté si cette dernière met en jeu la première personne du singulier du futur simple, du présent ou du passé simple de

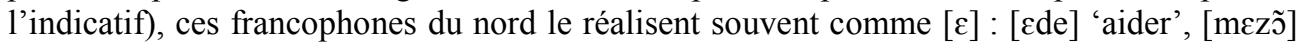

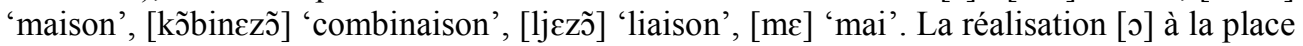
de $/ \mathrm{o} /$ chez les locuteurs du nord n'est pas toujours prévisible dans le sens où elle peut se produire en dehors du contexte attendu, c'est-à-dire en syllabe ouverte. Dans ce cas, on est

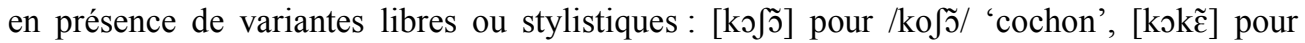
/kokẽ / 'coquin'.

\subsection{L'opposition $/ \tilde{\varepsilon} / \sim / \tilde{e} /$}

$\mathrm{Si}$ dans le parler français de certaines communautés francophones comme la France métropolitaine, il y a neutralisation de l'opposition $/ \tilde{\varepsilon} / \sim / \tilde{\mathfrak{e}} /$ ( $\tilde{\mathfrak{e}}$ ayant disparu au profit de

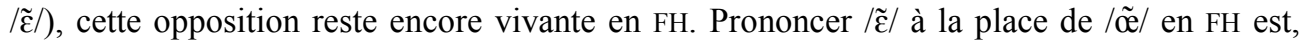
aux yeux des locuteurs haïtiens en général, un signe d'inculture ou celui d'un manque de maîtrise de la prononciation du français. L'Haïtien francophone, en s'exprimant en français, recourt à une variété livresque de français qu'il a apprise à l'école au contact des manuels

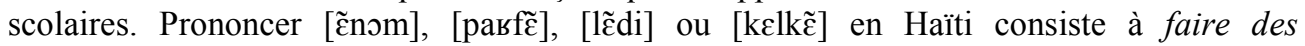
surettes c'est-à-dire mal prononcer un mot, ou pratiquer un français marron et, ainsi, se présenter comme possédant une maîtrise approximative du français. On doit toujours

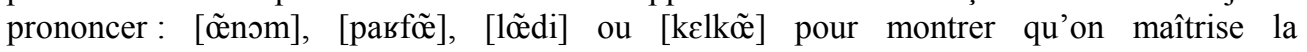

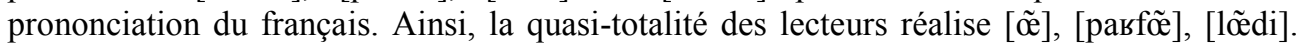
Un lecteur sur 10, docteur en sciences de l'éducation, dans la quarantaine, qui a étudié à

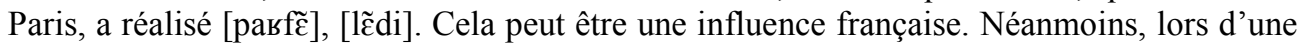
discussion informelle à la suite de la lecture, il a eu à réaliser à plusieurs reprises : [kelkõ] pour quelqu'un, [थ̃nom] pour un homme.

La neutralisation de l'opposition $/ \tilde{\varepsilon} / \sim / \tilde{\mathfrak{e}} /$ n'est pas très ancienne en français de la France continentale. Cette opposition y demeurait à une époque assez récente. Par exemple, selon des données de Martinet (1945), reprises par Avanzi (2017, cf. : https://francaisdenosregions.com/2016/12/18/2051/), une forte majorité de la population de la France métropolitaine (plus de 90\%) distinguait brin (comme dans brin d'herbe) et brun (comme dans ours brun). Avanzi (op. cit.) rappelle que des enquêtes récentes montrent que cette opposition connait une nette neutralisation en France continentale au profit de $/ \tilde{\varepsilon} /$ en soulignant qu'elle persiste encore dans le sud, notamment dans le SudOuest. Cependant, selon ces mêmes données, la distinction entre les deux voyelles se 
maintient encore normalement en Haïti (selon $84,3 \%$ des consultés) et en français antillais (67,1\% des consultés), se rapprochant ainsi du français québécois et, dans une moindre mesure, des variétés du sud de la France, de la Belgique et de la Suisse. Le /õ/ se conserve

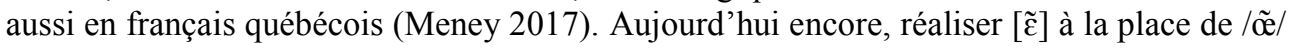
en FH revient à se faire étiqueter de pratiquer un français approximatif, ne respectant guère les normes de fonctionnement de la langue.

\subsection{L'opposition /a/ /a/}

L'opposition /a/ /a/ semble n'avoir jamais existé en $\mathrm{FH}$, ce qui fait que cette variété de parler francophone a toujours eu 15 voyelles contre les 16 qu'il y a eu en français de la France continentale qui aujourd'hui tend à en avoir 14 (avec la tombée en désuétude de /a/

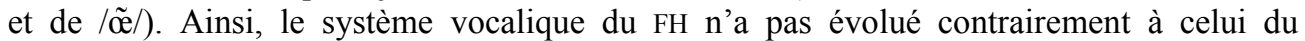
français de France par exemple. Mais cela ne veut pas dire que toutes les voyelles communes aux deux variétés sont réalisées de la même manière en FH qu'en FR. Une étude reste à faire là-dessus.

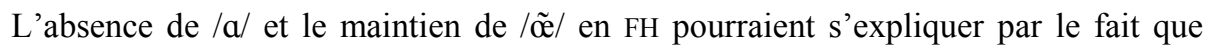
l'Haïtien s'approprie le français en situation scolaire au contact notamment du livre qui constitue aussi le contact immédiat de l'enseignant. Car, mis à part des cas où l' 'a' est surmonté du diacritique dit accent circonflexe, la graphie des sons [a] et [a] dans les variétés où existe l'opposition $/ \mathrm{a} / \sim / \mathrm{a} /$ est pareille. De même, que /õ/ se réalise comme $[\tilde{\varepsilon}]$ dans certaines variétés de français n'en change pas la graphie. Ainsi, l'écrit conventionnel en FR ne correspond pas toujours à la prononciation. Or, la langue évolue davantage à l'oral qu'à l'écrit, l'écrit étant une tentative de reproduction de l'oral à partir de signes graphiques conventionnels. Le manuel d'enseignement général de langue n'a guère la prétention de prendre en considération toutes les dimensions orales. Par exemple, beaucoup d'Haïtiens

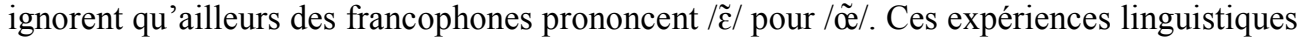
se manifestent dans le parler et non dans l'écrit. Ainsi, le manuel scolaire est souvent en décalage avec la pratique de la langue orale.

\section{4 À propos du schwa}

Le schwa renvoie à ce qu'on appelle généralement l'e caduc en français. L'e caduc est « tout E écrit sans accent orthographique en syllabe ouverte qui se prononce ou qui s'élide » (Léon, 2008 : 134). En FH en général, il y a prononciation et non élision du schwa à l'intérieur de mot. En fin de mot, en général, il ne se réalise pas. L'omission du schwa à l'intérieur d'un mot ou entre deux mots faisant partie d'un même syntagme en FH est vécu comme une marque de xénité. Sinon, le locuteur se fait accuser de zuzu (maniéré en parlant français) ou de petit Français. De même, pour l'énoncé «Je remercie mon neveu qui regarde passer les chevaux par la fenêtre. Celui-là a eu tellement peur qu'il a crié : " $\mathrm{Au}$ secours ! » au fur et à mesure que les chevaux passaient », la réalisation la plus récurrente dans les enregistrements est

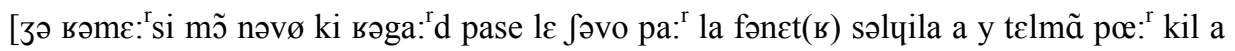

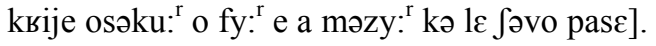

La majorité des lecteurs produisent cet énoncé en réalisant les schwas excepté le professeur d'anglais qui ne l'a pas réalisé dans les exemples [la skвetєь ... mõnvø ... la fnetь ... овkиь]. Un lecteur de 28 ans, journaliste de niveau de licence de communication et de linguistique, a réalisé [oskur].

Un lecteur de 65 ans (qui a étudié en France de la maîtrise au doctorat en sociologie) réalise souvent des schwas en fin de mot mais pas systématiquement :

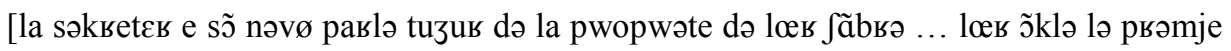

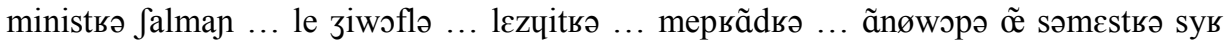




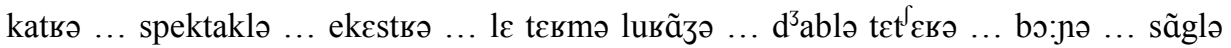
desãbьə vãdьə ... ьәьавdə pase ... la fənєtьə... о səkuь o fук е а тzуь ]

La réalisation du schwa en coda de syllabe facilite la réalisation des codas branchantes dont la prononciation est assez rare en $\mathrm{FH}$ parce que rare en $\mathrm{CH}$.

La récurrence de la réalisation du schwa en FH peut être liée au fait que le débit du FH est assez lent et l'économie dans ce cas passe au second plan. Elle peut aussi tenir au fait que le phénomène (dont la réalisation rappelle le registre soutenu hérité de l'apprentissage scolaire privilégiant en Haïti un français livresque) est absent du $\mathrm{CH}$. Néanmoins, les

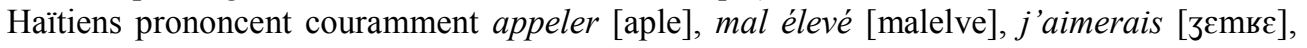
par exemple.

\section{L'assibilation}

Suivis de $/ \mathrm{i} /, / \mathrm{y} /, \mathrm{j} /$ et $/ \mathrm{u} /$, nous observons une légère assibilation de $/ \mathrm{t} / \mathrm{et} / \mathrm{d} /$ dans la pratique du FH en général. L'assibilation désigne la réalisation sous une forme chuintante ou sifflante, dans un contexte donné, d'une consonne occlusive. En FH, comme dans d'autres variétés de français, $/ \mathrm{t} / \mathrm{et} / \mathrm{d} /$ peuvent se réaliser sous une forme assibilée, c'est-à-dire plus ou moins affricativisée lorsqu'ils sont suivis de $/ \mathrm{i} /$ et $/ \mathrm{y} /$ ou des glides $/ \mathrm{j} /$ et $/ \mathrm{u} /$ en devenant respectivement $\left[\mathrm{t}^{\mathfrak{}}\right]$ et $\left[\mathrm{d}^{3}\right]$. Par exemple, l'énoncé

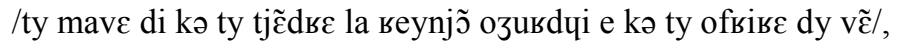

(Tu m'avais dit que tu tiendrais la réunion aujourd'hui et que tu offrirais du vin)

les locuteurs réalisent généralement

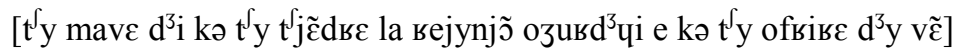

Le même phénomène s'observe aussi dans certains milieux de la France continentale dans les mêmes environnements (Candea 2017), entre autres dans les banlieues parisiennes où la plupart des locuteurs réalisent, par exemple, les mots $d u r$ et étudiant, comme [d $\mathrm{d}^{3} \mathrm{y}$ ] et $\left[\operatorname{et}^{\int} \mathrm{yd}^{3} \tilde{a}\right]$. Les mêmes consonnes, devant les mêmes voyelles et les mêmes glides, conduisent à une assibilation en français québécois (Côté 2014, Gendron 2014), mais elle est plus forte en français québécois qu'en FH.

Le professeur d'anglais ne fait pas d'assibilation dans lundi, diable, gratuit et produit. Mais on la sent dans sa lecture dans les mots friture, aujourd'hui, du, dit, têtière. En

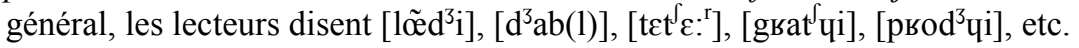

\section{Nasalisation de /t/}

/t/ précédé d'une $\tilde{V}$ et suivi d'une consonne non nasale peut être nasalisé. C'est le cas

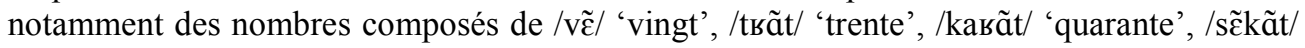
'cinquante', /swasãt/ 'soixante'. Les 10 lecteurs ont tous lu [dø mil vẽntwa] pour le nombre « 2023 » (où nous pouvons signaler l'amuïssement de / / / de /tıwa/). Cette expérience peut être commune à d'autres variétés de français.

\section{Nombre comportant $/ \mathbf{t} /$}

FR

$$
\begin{aligned}
& \text { [v } \tilde{\varepsilon} \mathrm{s} \tilde{\varepsilon} \mathrm{k}]>[\mathrm{v} \tilde{\varepsilon} \mathrm{ts} \tilde{\varepsilon} \mathrm{k}] \\
& \text { [tвãtset] } \\
& \text { [tвãtdø] }
\end{aligned}
$$

[kава̃ttıwa]

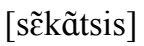

[swasãtdis]
FH

$$
\begin{aligned}
& \text { [vẽns ع̃k] } \\
& \text { [tвãnset] } \\
& \text { [tвãndø] }
\end{aligned}
$$

[swasãndis] 
Pour la vingtaine de 'quatre-vingt' (de 'quatre-vingt' à 'quatre-vingt-dix-neuf'), nous

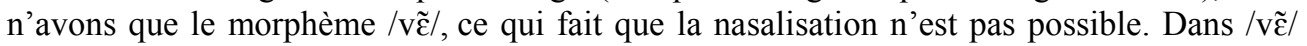
(vingt), il y a un [t] latent qui est susceptible de se prononcer dans certains contextes.

Cependant, si le $/ t /$ est suivi d'une voyelle ou d'une consonne nasale $(\tilde{C})$, la nasalisation n'a pas lieu. Ainsi, avec les unités 1, 8 et 9 ('un', 'huit' et 'neuf'), le /t/ ne se nasalise pas :

\begin{tabular}{|c|c|c|}
\hline Nombre & FR & FH \\
\hline $30,40,60$ & 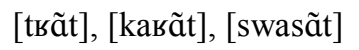 & [tва̃t], [кака̃ $]$, [swasãt] \\
\hline 31 & [tвãteõ] & [нва̃tejœ̃ ] \\
\hline 48 & [kавãtıit] & [кавãtuit] \\
\hline 29 & [vẽtnœf] & [vẽtncf] \\
\hline 71 & [swasãte õz] & [swasãn õz] \\
\hline
\end{tabular}

Si en FR on dit /swasãte õz/, en FH on dit /swasãt õz/. C'est pour cette raison qu'on observe la nasalisation de /t/ confirmant ainsi qu'il y a une frontière morphémique entre le

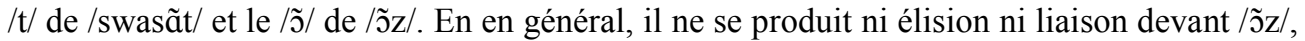
ce qui fait penser à l'existence d'une consonne latente au début du mot /õz/ dans la représentation phonologique des locuteurs.

Avec /nœf/, on pourrait s'attendre à une nasalisation de /t/ qui pourrait se traduire par une gémination de $/ \mathrm{n} /$. Mais rien de tel ne se produit. Cette non-nasalisation pourrait s'expliquer par le non-voisement de $/ \mathrm{t} /$ et par le fait que $/ \mathrm{t} /$ et $/ \mathrm{n} /$ partagent le même point d'articulation.

\section{Conclusion : influence du $\mathrm{CH}$}

Comme nous l'avons déjà souligné, tous les phénomènes phonétiques exposés ici ne sont pas des spécificités exclusives du FH. Ils ne relèvent certes pas tous du FR le plus commun mais peuvent être repérés dans la plupart des autres variétés de parlers francophones. Ils ne sont certes guère observés chez tous les francophones, mais font partie d'une certaine grammaire phonologique du français en général où ils n'appartiennent pas à un registre socialement valorisé et reconnu comme tel. C'est pour cela qu'ils ne se produisent guère dans le discours surveillé. Néanmoins, certains d'entre eux, comme la nasalisation de /t/ dans les conditions élucidées, la réalisation labiale de /ь/ devant une voyelle labiale et son affaiblissement ou son amuïssement en contexte postvocalique, l'assibilation de $/ \mathrm{t} / \mathrm{et} / \mathrm{d} /$ suivis de /i, y, j, y/, le maintien de l'opposition $/ \tilde{\varepsilon} / \sim / \tilde{\mathfrak{e}} /$, l'expérience antihiatique dans le cas où deux voyelles se suivent dans un mot, la non-réalisation des codas branchantes finales, la réalisation de $/ \mathrm{h} /$ dit aspiré, la réalisation de $/ \mathrm{n} /$ sous la forme de [j] ou [nj], etc. semblent faire partie de la grammaire phonologique du FH. Ainsi, les variétés d'une langue commune peuvent certes partager un maximum de faits linguistiques communs, néanmoins, elles fonctionnent comme des systèmes linguistiques comportant des mécanismes ou des dynamiques de fonctionnement internes, ce qui fait que certains faits linguistiques peuvent être remarqués dans certaines et non dans d'autres.

Ainsi, tous les phénomènes étudiés n'ont pas lieu sous l'influence du CH. D'autant que certaines expériences phonologiques orales tels l'effacement des consonnes branchantes, l'affaiblissement voire la disparition de /b/postvocalique, la nasalisation de /t/ telle que présentée ou encore l'assibilation de /t/ et /d/ suivis de /i, y, j, y/ (le CH ne retenant pas la voyelle labiale antérieure /y/), rapprochent le $\mathrm{FH}$ du $\mathrm{CH}$ qui y emprime la plupart de ses marques. Les seules codas branchantes en $\mathrm{CH}$ se forment avec $/ \mathrm{ks} / \mathrm{et} / \mathrm{ps} /$ comme dans /taks/, /fiks/, /boks/, /tcks/, /klips/, etc. Ainsi, la récurrence de la non-réalisation des codas 
branchantes en FH semble être une influence du CH, même si elle est aussi constatée dans d'autres variétés de de parlers francophones. En outre, il existe une règle phonologique du $\mathrm{CH}$ selon laquelle aucune consonne occlusive orale voisée ne vient à la suite d'une $\tilde{\mathrm{V}}$ notamment en finale de mot. Elle est automatiquement nasalisée par la $\tilde{V}$ qui précède. C'est ce qui explique la tendance en $\mathrm{FH}$ à une nasalisation dans les mêmes conditions comme expliqué dans la section 2.3 avec la nasalisation des occlusives $/ \mathrm{b}, \mathrm{d}, \mathrm{g} /$. Cependant, en position médiane, il n'y a pas de nasalisation : [vãn] / [vãdab] 'vendre / vendable', [bõm] /

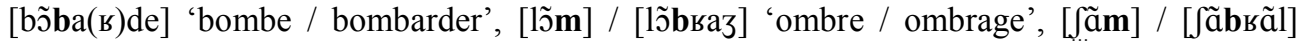
'chambre / chambranle'. La totalité des lecteurs a réalisé [kатекаmãn] iii 'caméraman', [wa/intõn] ] 'Washington', [tenismẽn] 'tennismen'. Un lecteur qui a étudié à Paris a réalisé [kameкаman]. Cela peut être une influence française. 9 des lecteurs ont renforcé le $/ \mathrm{p} / \mathrm{de}$ camping par un [m] : [kãmpĩn] après l'avoir nasalisé de manière progressive par la $\tilde{\mathrm{V}} / \tilde{\mathrm{a}} /$. Cependant, le lecteur de 65 ans a réalisé [g] après la vélaire finale : [kãmpĩng].

L'intervention d'une consonne épenthétique entre les deux voyelles contiguës d'un mot (pouvant être $/ \mathrm{j} /, / \mathrm{w} / \mathrm{ou} / \mathrm{s} /$ ) tient à un principe phonologique en $\mathrm{CH}$ qui se traduit par une règle graphique adoptée dans l'orthographe $\mathrm{du} \mathrm{CH}$, à savoir que deux voyelles ne se retrouvent pas de manière contiguë dans un mot en $\mathrm{CH}$ : elles sont séparées soit pas $/ \mathrm{j} /$, soit par $/ \mathrm{w} /$ selon que l'une d'entre elles est labiale. Ce phénomène antihiatique observé en FH est systématique en $\mathrm{CH}$ - langue première des francophones haïtiens, existe aussi dans d'autres variétés de français. On pourrait en dire autant pour la nasalisation de /t/ dans les numéros cardinaux, que nous avons étudiée.

\section{Références bibliographiques}

Akpossan, J. (2015). La consonne /R/ comme indice de la variation lectale : cas du français guadeloupéen en contact avec le créole guadeloupéen. Thèse de doctorat, Université Sorbonne Nouvelle - Paris 3.

Avanzi, M. (2017). Atlas du français de nos régions. Paris : Armand Colin.

Candea, M. (2017). Pratiques de prononciation et enjeux sociaux. Approches postvariationnistes en sociophonétique $d u$ français de France. Grenoble: Université Grenoble Alpes, https://hal.archives-ouvertes.fr/tel-01910667/document.

Côté, M.-H. (2014). «Liaison et assibilation en français laurentien ». In : Soum-Favaro C., A. Coquillon \& J.-P. Chevrot, La liaison : approches contemporaines. Berlin : Peter Lang, 9-31.

Darcy, I. (2006). Assimilation phonologique et reconnaissance des mots. Berne, Berlin, Bruxelles : Peter Lang (Col. Publications Universitaires Européennes, Série XXI, N ${ }^{\circ}$ 292.

Fattier, D. (2010). «Le français d'Haïti (dans sa relation osmotique avec le créole) : remarques à propos des sources existantes ». In: Thibault, A. (éd.), Le français régional antillais : exploration et délimitation d'un concept. Paris : L'Harmattan, 315337.

Gadet F. (2007). La variation sociale en français. Paris : Ophrys.

Gendron, J.-D. (2014). La modernisation de l'accent québécois, de l'accent traditionnel au nouvel accent, 1841-1960 : esquisse historique : contribution à l'histoire de la prononciation du français au Québec. Québec : Presses de l'Université Laval.

Govain, R. (2013). «Le français haïtien et l'expansion du français en Amérique ». In : Castellotti V. (dir.), Le(s) français dans la mondialisation. Bruxelles / Fernelmont: Éditions EME Intercommunications, 85-103.

Govain, R. (2009). Plurilinguisme, pratique du français et appropriation de connaissances en contexte universitaire en Haïti. Thèse de doctorat, Université Paris VIII.

GovaIN, R. (2008), Normes endogènes et enseignement-apprentissage du français en Haïti. Études créoles, $n^{o s} 1$ et 2, 131-164.

Hibbert, F. (1993) [1923]. Les simulacres. L'aventure de M. Hellenus Canton. Port-au- 
Prince : Éditions Fardin.

Léon, P. et al. (2008). Phonétique du FLE. Prononciation : de la lettre au son. Paris : Armand Colin.

Martinet, A. (1945). La prononciation du français contemporain, témoignages recueillis dans un camp d'officiers prisonniers. Paris/Genève : Droz.

Meney, L. (2017). Le français québécois entre réalité et idéologie. Un autre regard sur la langue. Étude sociolinguistique. Québec : Presses de l'Université Laval.

Navarro, S. (2016). Le / $r /$ en anglais. Histoire, phonologie et variation. Dijon : Éditions universitaires de Dijon.

Pierret, J.-M. (1994). Phonétique historique du français et notions de phonétique générale. Louvain-la-Neuve : Peeters (Nouvelle édition).

Pompilus, P. (1961). La langue française en Haïti. Thèse de doctorat, IHEAL.

Saint-Fort, H. (2007). «La langue de Jacques Roumain. Le 'français haïtien' dans "Gouverneurs de la rosée" : Analyse et classement des particularités lexicales du roman ». In : Leconte F.-A. (dir.), Haïti et Littérature. Jacques Roumain au pluriel. Paris : Books, 184-205.

Walker, D.C. (1984). The Pronunciation of Canadian French. Ottawa : University of Ottawa Press.

Warnant, L. (1996). Orthographe et prononciation en français : les 12.000 mots qui ne se prononcent pas comme ils s'écrivent. Louvain-la-Neuve : Duculot.

Je remercie l'évaluateur de la proposition, qui m'a permis d'enrichir la présentation. Mais je suis le seul responsable de toute erreur qui se serait glissée dans le texte.

\section{Annexe : Test de lecture sur la phonologique du français haïtien}

La secrétaire et son neveu parlent toujours de la propreté de leur chambre. Leur oncle, le premier ministre Charlemagne, aime le fromage, le girofle, la friture, les huitres, mais hait la palabre et se méprendre.

Il part en Europe un semestre sur quatre pour assister à des spectacles, faire du camping et du sport équestre. Il a récemment dit à son ami Salagnac : «Tu m'avais dit que tu tiendrais la réunion aujourd'hui et que tu offrirais du vin ».

Les termes noël, louange, Canaan, dehors, lundi, parfum, diable, têtière, gratuit, produit, seigneur, borgne, hache, trotter, croquer, caméraman, Washington, tennismen, sangle, décembre, vendre, association, prononciation ... existent aussi en créole.

Je remercie mon neveu qui regarde passer les chevaux par la fenêtre. Celui-là a eu tellement peur qu'il a crié : «Au secours ! » au fur et à mesure que les chevaux passaient.

Pour finir, j'aimerais qu'on arrive à enlever l'épreuve d'espagnol du concours à partir de 2023. Ce serait ni plus ni moins que juste notamment pour les nombreux candidats provenant d'écoles défavorisées qui cherchent ardemment une place à l'Université d'État d'Haïti.

\footnotetext{
${ }^{\mathrm{i}}$ Ces deux derniers exemples ont été produits en chœur par une classe de seconde d'une école « congréganiste », c'est-à-dire une école dirigée par une congrégation catholique au Cap-Haïtien où j'ai été invité à prononcer une conférence sur la francophonie en 2018.
} 
${ }^{i i}$ Il est à noter qu'un lecteur de 62 ans enseignant d'anglais a réalisé un 'h' très 'aspiré' : [dəhəь].

iii Un lecteur doctorant en science du langage de 43 ans a réalisé [катеваmẽn].

${ }^{\text {iv }}$ Un professeur d'anglais de 62 ans a réalisé [wafintən] comme à l'américaine. 